\title{
Towards improved humidity measurements at high temperatures and transient conditions
}

\author{
Martti Heinonen ${ }^{1}$, Fausto Arpino ${ }^{2}$, Rien Bosma ${ }^{3}$, Laura Cavallarin ${ }^{4}$, Gino Cortellessa ${ }^{2}$, Marco Dell' Isola ${ }^{2}$, Volker Ebert ${ }^{5}$, \\ Vito C. Fernicola ${ }^{6}$, Eric Georgin ${ }^{7}$, Antonio Giannattasio ${ }^{8}$, Richard Högström ${ }^{1}$, Domen Hudoklin ${ }^{9}$, Anders Kentved ${ }^{10}, J_{a n}$ \\ Nielsen ${ }^{11}$, Peter Friis Østergaard ${ }^{11}$, Andrea Peruzzi ${ }^{3}$, Tomi Pietari ${ }^{12}$, Robert-Jan Pouw ${ }^{13}$, Shahin Tabandeh ${ }^{14}$, Wilhelm van \\ Schaik $^{15}$ and Steven Wagner ${ }^{16}$
}

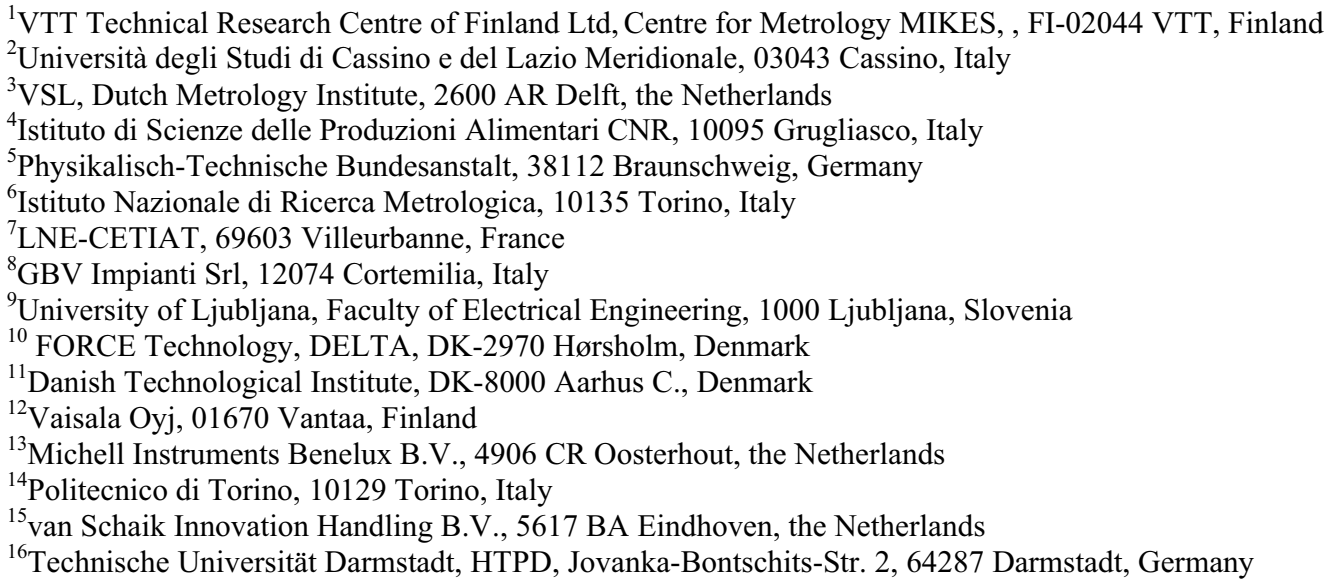

\begin{abstract}
Humidity is a key parameter in controlling drying processes and ambient conditions in many industrial manufacturing, storage and test applications. Air humidity is routinely measured at temperatures above $100^{\circ} \mathrm{C}$ and at conditions that are often challenging due to temporal and local variations. Calibrations of humidity sensors do not provide appropriate representativeness of measurement conditions because they are limited to temperatures below $100^{\circ} \mathrm{C}$ and static conditions. A European metrology research project HIT ("Metrology for Humidity at High Temperatures and Transient conditions") is developing improved humidity measurement and calibration techniques to temperatures up to $180{ }^{\circ} \mathrm{C}$ and non-static conditions. This paper summaries developments of the project: calibration and test facilities for industrial hygrometers, studies on humidity control in specific microbial transient processes and a new measurement approach for water activity measurements.
\end{abstract}

\section{Introduction}

Drying is a part of most manufacturing processes. Optimising drying processes through improved humidity control has significant potential to reduce the energy consumption and waste production. In industrial dryers, the humidity of the high temperature exhaust air/gas (typically around $140{ }^{\circ} \mathrm{C}$ but can be up to $180{ }^{\circ} \mathrm{C}$ depending on the process) is measured. Ideally, higher inlet air temperatures reduce the process time but require careful and fast in-line temperature humidity vs. time control for early detection of the end-point in order to prevent deterioration of the material exiting the dryer. Similar process control challenges are associated with drying/baking in food processing, drug manufacturing, paper production, ceramic production, wood processing and textile production. However, humidity calibrations are not usually performed at temperatures above $100{ }^{\circ} \mathrm{C}$ and the calibration equipment that is commercially available for industry cannot be operated in this range.

Non-static conditions in processes and environmental tests form another type of challenge because different humidity sensors have different response times. Temporal and spatial variations of humidity in a process line make it also impossible to measure water activity, i.e. equilibrium relative humidity, directly although this is an important parameter in food processing.

* Corresponding author: author@e-mail.org

(C) The Authors, published by EDP Sciences. This is an open access article distributed under the terms of the Creative Commons Attribution License 4.0 (http://creativecommons.org/licenses/by/4.0/). 
These problems in industrial humidity measurements are addressed by the HIT project (14IND11 "Metrology for Humidity at High Temperatures and Transient conditions") funded by the European Metrology Programme for Innovation and Research (EMPIR). The project has been running since September 2015 and it will end in 2018. The project consortium is composed by 15 partner organisations. Further information about the project is available in [1] and at www.empir-hit.eu.

This paper summarises advancements in the HIT project: calibration and test facilities for industrial hygrometers, tests for developing new calibration procedures, studies on humidity control in specific microbial transient processes and a new measurement approach for water activity measurements.

\section{Calibration and test facilities}

New calibration facilities have been developed and existing systems have been modified to provide a robust SI traceability link and versatile calibration and testing environments. These will be used for defining optimal calibration procedures covering also temperatures above $100{ }^{\circ} \mathrm{C}$ and non-static conditions.

\subsection{A new humidity generator based on indirect gravimetric approach}

A new generation humidity standard has been developed at Istituto Nazionale di Ricerca Metrologica (INRIM, Italy). It is founded on a mass fraction vapour generator capable to operate at dew-point temperatures above $100^{\circ} \mathrm{C}$. The operation of the generator is based on indirect gravimetric approach, i.e. the water mass fraction of outlet air is controlled by SI traceable mixing of a water mass flow evaporated into a dry air. This provides SI-traceability for humidity measurements at temperatures up to $180{ }^{\circ} \mathrm{C}$, dew-point temperatures up to $140{ }^{\circ} \mathrm{C}$ and pressures up to $0.6 \mathrm{MPa}$ (abs) [2]. In terms of absolute humidity, the generator operates over a range from $25 \mathrm{~g} / \mathrm{m}^{3}$ at atmospheric pressure to approximately $1000 \mathrm{~g} / \mathrm{m}^{3}$ at $0.6 \mathrm{MPa}$ (abs). A microwave resonator is also integrated in the system as a reference. The operation principle of the generator system is illustrated in Fig. 1.

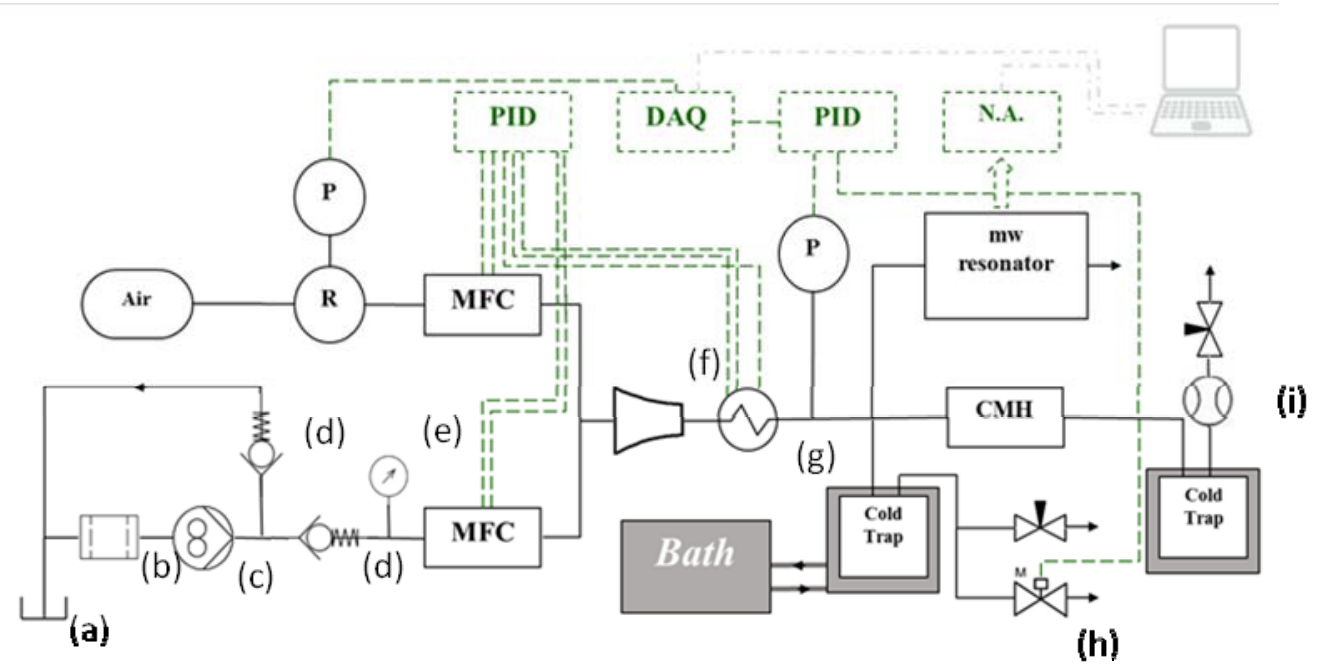

Fig. 1. Schematic diagram of the INRIM humidity generator system. (a) Water reservoir, (b) Filter.(c) Gear pump, (d) Check valve, (e) Barometer, (f) Mixing nozzle, (g) Heat exchanger, (h) Solenoid valve, (i) Mass Flow Meter, (P) Pressure gage, (R), Pressure regulator, $(\mathrm{CMH})$ Chilled Mirror Hygrometer, (DAQ) Data Acquisition Unit, (MFC) Mass Flow Controller (N.A.) Network Analyzer.

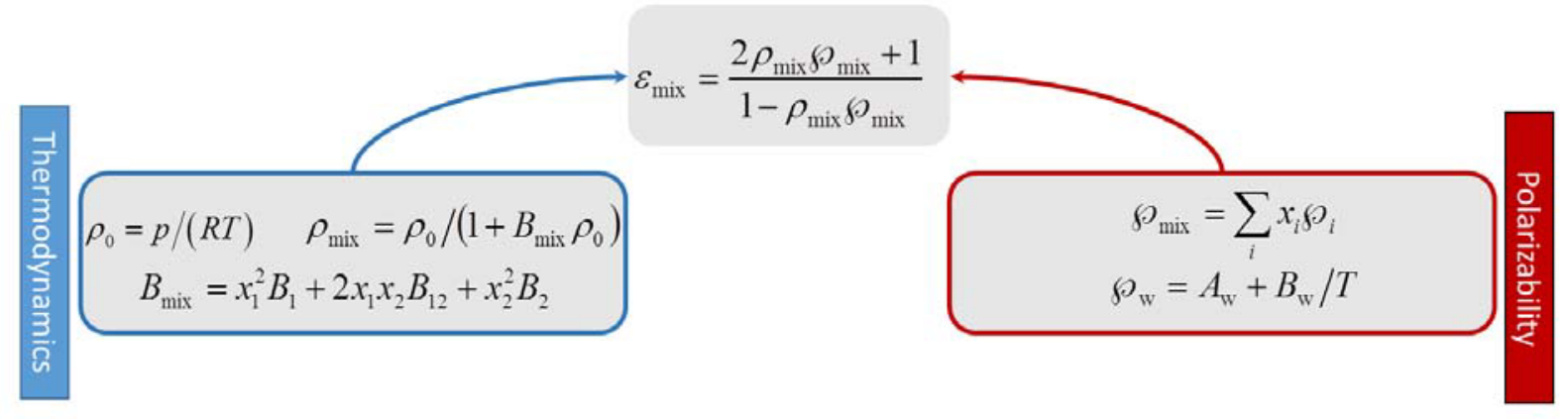

Fig. 2. Governing equations for microwave hygrometry. Symbols are described in Table 1. 
Table 1. Symbols of the governing equations for microwave hygrometry in Fig 2.

\begin{tabular}{|l|}
\hline$\varepsilon_{\text {mix }}=$ Permittivity of the mixture \\
\hline$\rho_{\text {mix }}=$ Polarisabity of the mixture \\
\hline$\rho_{\text {mix }}=$ Molar density of the mixture \\
\hline$R=$ Molar gas constant \\
\hline$T=$ Temperature \\
\hline$P=$ Pressure \\
\hline$B_{\text {mix }}=$ Second density virial coefficient of the mixture \\
\hline$x_{\mathrm{i}}=$ Corresponding mole fraction \\
\hline $\begin{array}{l}B_{1}=\text { Second virial coefficient (interactions of water } \\
\text { molecules) }\end{array}$ \\
\hline $\begin{array}{l}B_{12}=\text { Second cross virial coefficient (interactions of } \\
\text { nitrogen and water molecules) }\end{array}$ \\
\hline $\begin{array}{l}B_{2}=\text { second virial coefficient (interactions of nitrogen } \\
\text { molecules) }\end{array}$ \\
\hline$\wp_{\mathrm{i}}=$ molar polarizability of pure component $i$ \\
\hline$A_{\mathrm{w}}$ and $B_{\mathrm{w}}$ : Debye constants \\
\hline
\end{tabular}

The vapour generator has been validated through absolute mole fraction measurements in the gas phase, by exploiting microwave-based hygrometry and by comparison against a primary humidity standard in the relevant dew-point sub-range [3]. Microwave hygrometry is a well-known method able to detect the vapour mole fraction of binary mixtures with a high precision using the cavity microwave resonance frequencies. The permittivity of the mixture could be calculated using the resonance frequencies and is connected to the density in one hand and polarizability of the mixture on the other hand (see Fig. 2). The simultaneous solution of aforementioned equations gives the water to gas mole fraction which exhibits a very high sensitivity due to the high polarizability of water and is well suited to cover the vapour amount fraction from $0.04 \mathrm{~mol} / \mathrm{mol}$ to $0.6 \mathrm{~mol} / \mathrm{mol}$ which matches the generator range.

The water vapour mole fraction, as measured by the microwave hygrometer, and the specific humidity, as generated by the mass fraction vapour generator, were compared over a wide range of pressure and temperature. Fig. 3 shows the water vapour mole fraction, as measured by the microwave hygrometer in blue, and the equivalent specific humidity, as generated by the mass fraction vapour generator in orange. In this range, the relative uncertainty of the microwave hygrometer is about one percent.

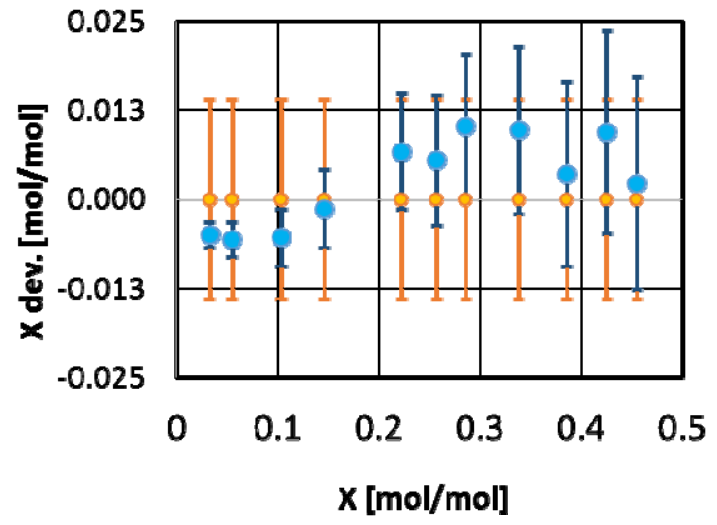

Fig. 3. Comparison of the microwave hygrometer (in blue) and the generator (in orange) at INRIM in terms of water mole fraction difference. The generator was used as the reference.

\subsection{A new modular humidity calibration setup}

For improving the efficiency of humidity calibrations, VTT MIKES has developed a modular humidity calibration setup that can easily be installed any commercial temperature test chamber with inner volume of $200 \mathrm{dm}^{3}$ or larger. In this setup, the humidity in a measurement chamber is controlled by two thermal mass flow controllers located outside the temperature test chamber. These controllers are connected to the measurement chamber system with flexible tubes equipped with quick couplings. This enables easy assembling and disassembling of the system. A LabView computer program was developed for enabling automatic calibrations in static and non-static conditions. The schematic diagram of the system is in Fig. 4.

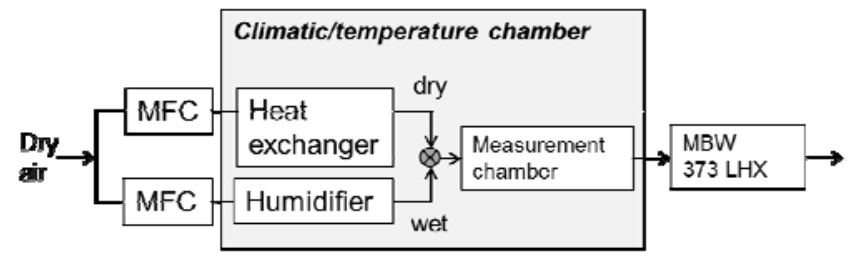

Fig. 4. Schematic diagram of the modular humidity calibration setup developed at VTT MIKES.

An MBW373 LHX chilled mirror hygrometer is used as the dew-point temperature reference. The air temperature in the measurement chamber is measured with two Pt100 thermometer probes. One of the probes is in a metal tube through which air flows to the reference dew-point hygrometer. This is located in the middle of the chamber (see Fig. 5) while the other thermometer probe is close to rear and bottom walls of the chamber. A fan is circulating air in the chamber to reduce humidity gradients when operating in non-static conditions. The inner volume of the measurement chamber is $12 \mathrm{dm}^{3}$. 


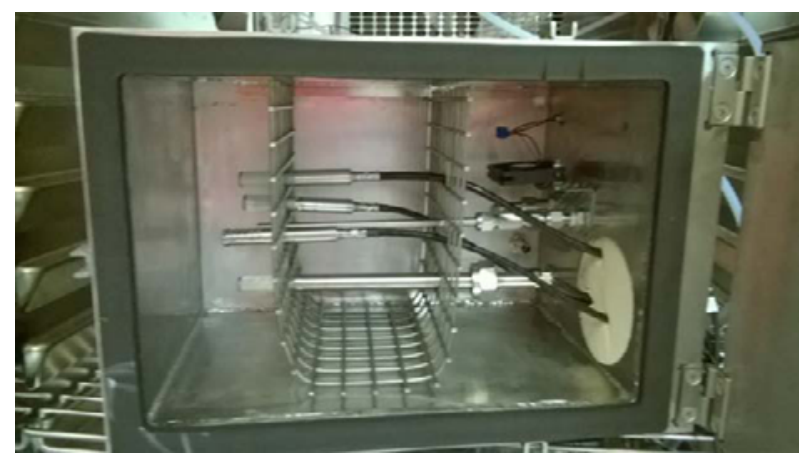

Fig. 5. Photograph showing humidity probes in the measurement chamber of the VTT MIKES calibration setup. The metal outlet tube is in the middle.

As the system is operated with an input flow rate of $2 \mathrm{l} / \mathrm{min}$, the inner volume of the chamber limits the speed of humidity change, which can be seen as increased difference between the set point and the measured humidity in Fig. 6. To enable measurements with faster humidity changes, an optional feeding tube arrangement was developed. As illustrated in Fig. 7, in this configuration humidity controlled air is split into two channels in the chamber: one for the reference dew-point hygrometer and the other one for the humidity probe under test.

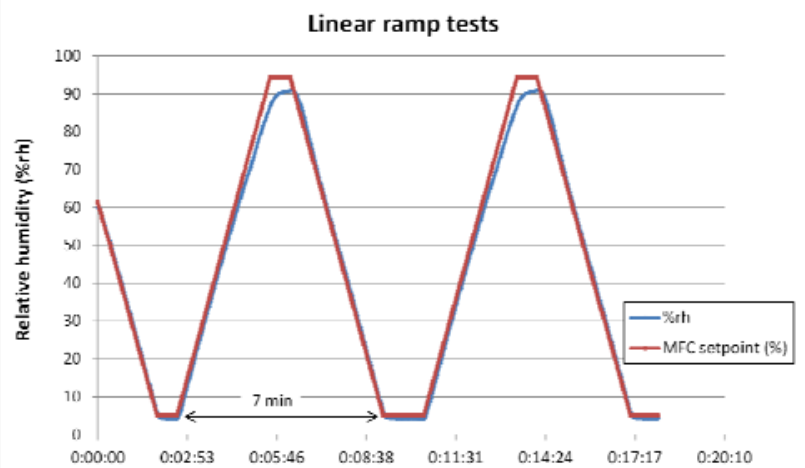

Fig. 6. Test results showing the actual relative humidity with respect to the set point when running linear ramps in the VTT MIKES calibration setup.

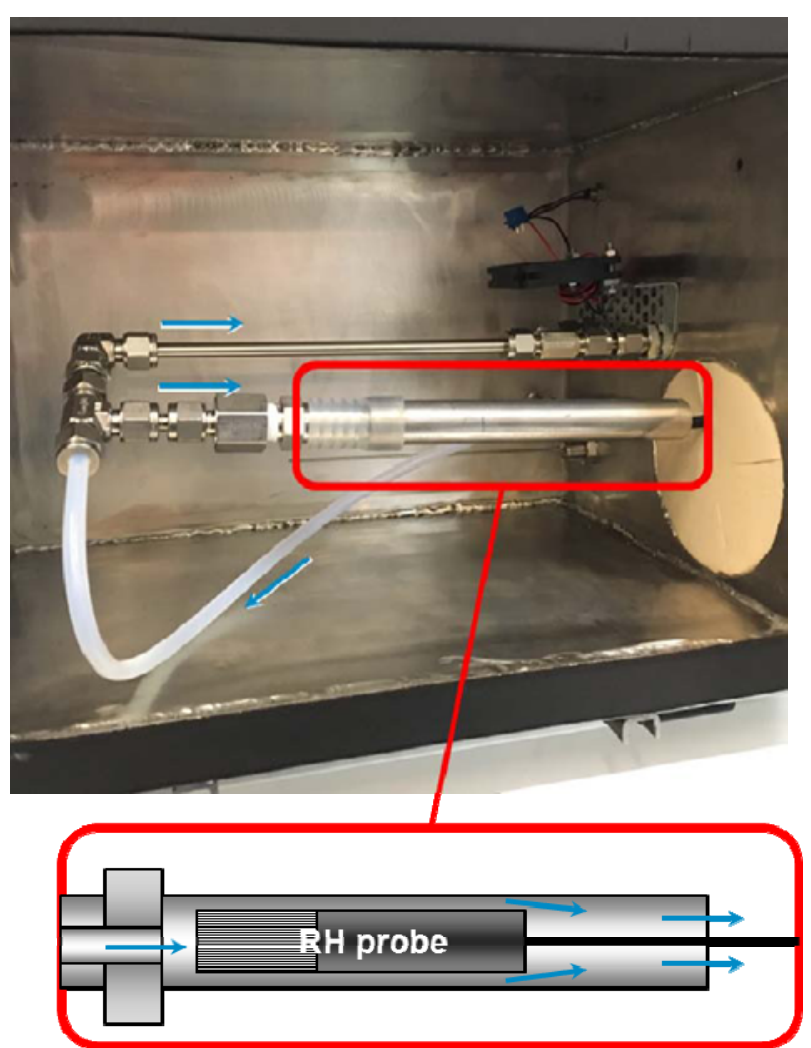

Fig. 7. Measurement configuration for tests with fast humidity changes (e.g. step changes) in the VTT MIKES calibration setup.

\subsection{Extending the operation range of a humidity generator}

A humidity calibration system at CETIAT comprises a saturator immersed in a liquid bath and a measurement chamber located in a thermostatic chamber (Fig. 8). To enable relative humidity calibrations at temperatures up to $140{ }^{\circ} \mathrm{C}$, CETIAT has extended the operation range of the humidity calibration system by developing new temperature control of the tubing between the saturator and the measurement chamber and by performing a thermal characterisation of the measurement chamber in the full temperature range.

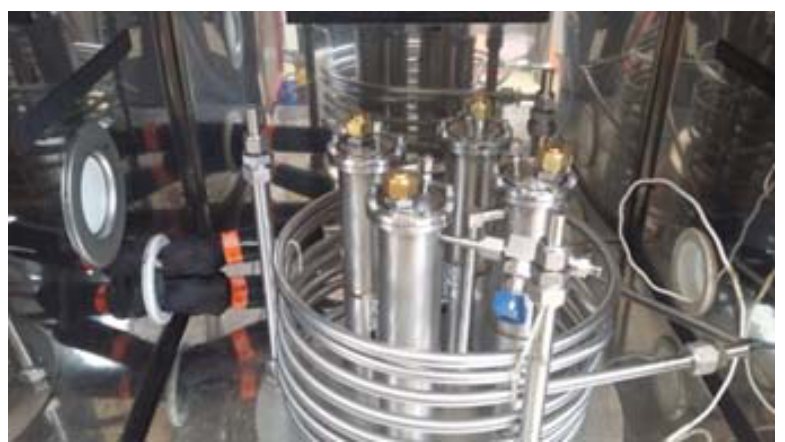

Fig. 8. Measurement chamber of the CETIAT humidity generator. 
Performed tests indicate that the uncertainty of $0.06{ }^{\circ} \mathrm{C}$ $(k=2)$ assigned for the range up to $80^{\circ} \mathrm{C}$ is also achieved in the extended dew-point temperature range from $80{ }^{\circ} \mathrm{C}$ to $90{ }^{\circ} \mathrm{C}$. Temperature differences in the measurement chamber were found to be within $\pm 0.2^{\circ} \mathrm{C}$ (see Fig. 9).

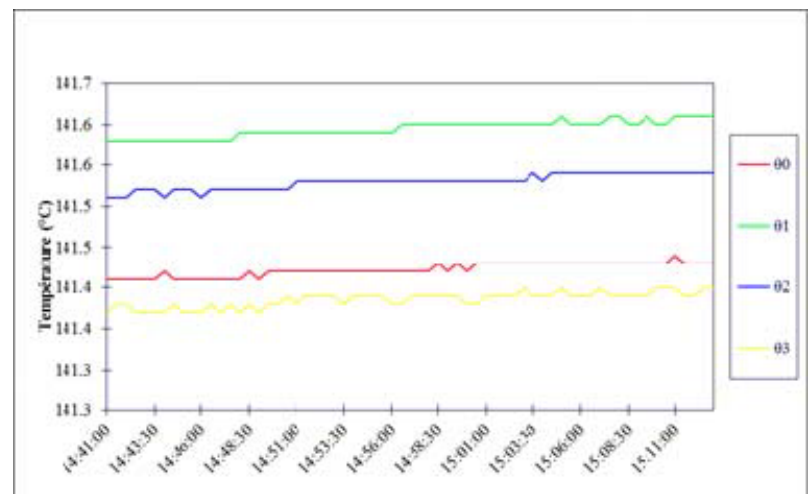

Fig. 9. Example of results obtained in the thermal characterisation of the CETIAT measurement chamber. The uncertainty of the temperature measurements was $0.2^{\circ} \mathrm{C}(k=2)$.

\section{Tests for developing new humidity calibration procedures}

The calibration and test facilities described above and a humidity calibration facility of VSL operating up to $180^{\circ} \mathrm{C}$ and $0.6 \mathrm{MPa}[4,5]$ are being used to develop calibration procedures that will effectively disseminate SI traceability to humidity measurements at high temperatures, high pressures and non-static conditions. For this purpose, several types of humidity sensors are being tested at static conditions in a wide range of temperature and pressure and at ambient pressure level with different humidity ramps. The aim is to identify most practical procedures for use in industrial calibration laboratories. Aspects to be considered are validity of calibration results to measurement conditions, amount of additional work due to the extended temperature range and cost of investment to calibration equipment.

In addition to commercially available sensors, tests have been carried out for prototype sensors: Three Michell prototype sensors (Fig. 10) were tested at temperatures up to $180^{\circ} \mathrm{C}$ and pressures up to $550 \mathrm{kPa}$ (dew-point temperatures up to $144{ }^{\circ} \mathrm{C}$ ). The results of the tests were used to apply temperature-dependent corrections to the relative humidity indicated by the sensors and to investigate the sensors aging. Also, two IH acoustic prototype sensors were studied at VSL (Fig. 11). The first one was tested at temperatures up to $100{ }^{\circ} \mathrm{C}$ and atmospheric pressure to obtain the temperature dependence of the speed of sound. To study the pressure dependence, measurements were carried out with the second prototype at temperatures up to $120^{\circ} \mathrm{C}$ and pressures up to $500 \mathrm{kPa}$.

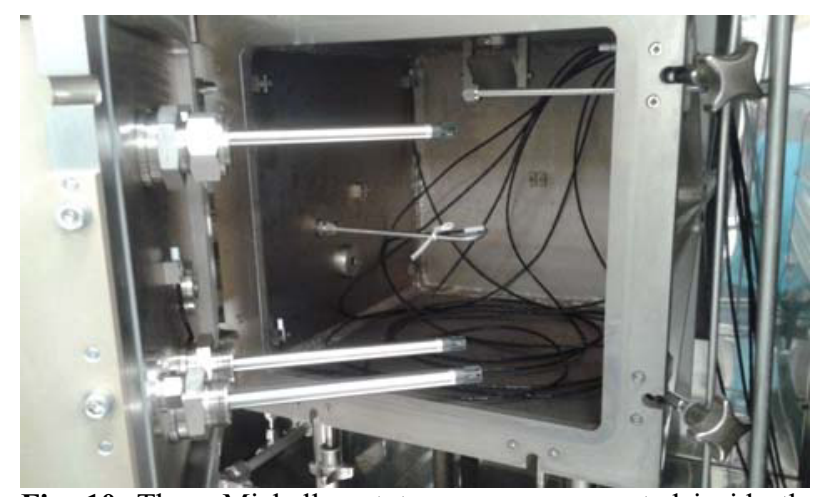

Fig. 10. Three Michell prototype sensors mounted inside the VSL chamber. Tests were carried out at temperatures up to $180^{\circ} \mathrm{C}$ and pressures up to $550 \mathrm{kPa}$.

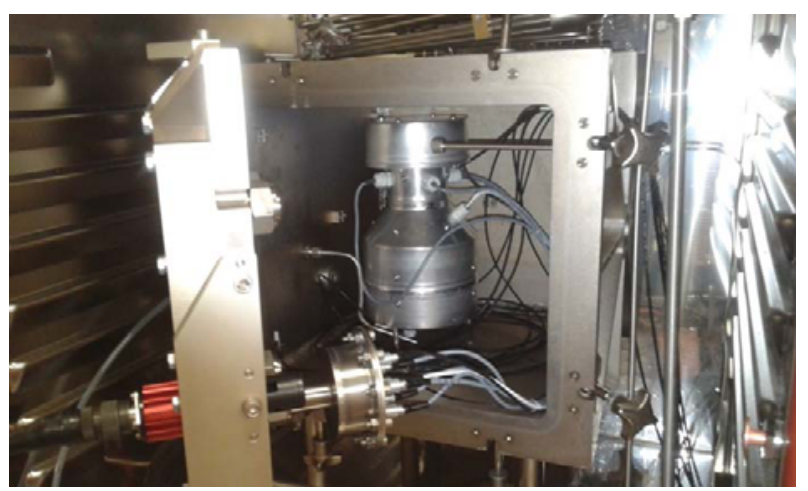

Fig. 11. IH acoustic prototype sensor accommodated inside the VSL chamber.

\section{A new approach for inline water activity measurement}

Water activity is an important parameter in food and feed processing. It equals to the relative humidity of air (expressed on a scale of 0 to 1 ) having no net exchange of water with the substance of interest. For improving cost efficiency and product quality in industrial processing, a reliable method for inline determination of water activity is needed. A new approach was developed by combining traceable continuous temperature and sample-based sorption isotherm measurements and appropriate new simulation tools.

\subsection{Development of sorption isotherm measurement methods}

For studying the approach, Università degli Studi di Cassino e del Lazio Meridionale (UNICAS) realized an experimental setup for the determination of sorption isotherms - i.e. the relationships between water activity and moisture content - and performed initial measurements with hazelnuts in the temperature range of $20{ }^{\circ} \mathrm{C}$ to $60{ }^{\circ} \mathrm{C}$. In the setup, hazelnut samples were at first stabilised in humidity controlled enclosure (glass jar with saturated salt solutions) and then measured with a commercial moisture balance. The equilibrium conditions were measured with a chilled mirror hygrometer and a Pt100 thermometer. Fig. 12 shows an example of obtained results. 


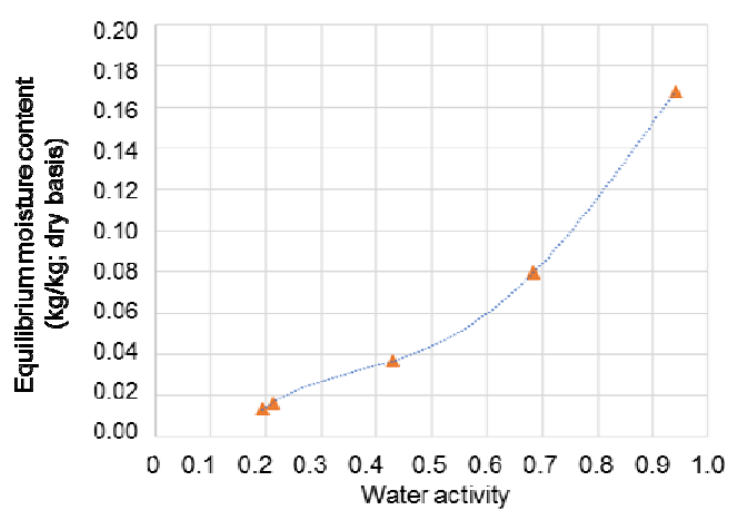

Fig. 12. Adsorption isotherm of 10 hazelnuts at $30^{\circ} \mathrm{C}$.

A new and more advanced system for sorption isotherm measurements was developed at the Danish Technological Institute (DTI). As illustrated in Fig. 13, the system is comprised of a saturator, a sample chamber, an oven, a scale, and a dew-point hygrometer. Air is pumped through the saturator submerged in a temperature controlled liquid bath. After complete saturation with respect to water, the air flows to the sample chamber inside the oven. In the sample chamber, the air flows through the sample. If the sample is not in equilibrium with the inlet air, net absorption or desorption will take place. Two independent systems detect the absorption or desorption of water in the sample. The entire sample chamber is placed on top of a scale which registers any change in weight due to a change in the water content of the sample. The dewpoint hygrometer placed after the oven provides the other method for detecting if the sample is in equilibrium with the inlet air. Once the sample is in equilibrium, the sample temperature, dew-point temperature and sample weight are recorded. Sorption isotherms are determined by combining these results obtained at different temperatures and humidities.

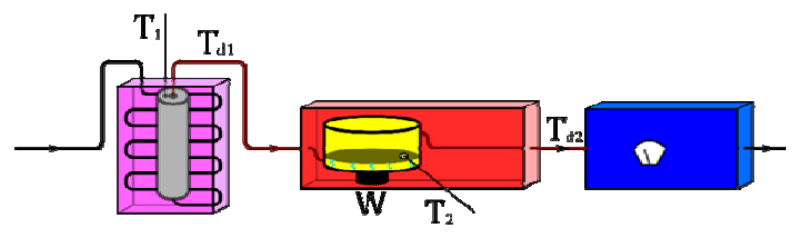

Fig. 13. Schematic diagram of the sorption isotherm measurement systems developed at DTI. $\mathrm{T}_{1}=$ saturator temperature, $\mathrm{T}_{\mathrm{d} 1}=\mathrm{dew}$-point temperature at the saturator outlet, $\mathrm{T}_{2}=$ air temperature in sample chamber, $\mathrm{T}_{\mathrm{d} 2}=$ dew-point temperature at the hygrometer inlet, $\mathrm{W}=$ weight of the sample continuously monitored by a scale.

\subsection{Development of simulation tools}

UNICAS developed a transient tool for modelling temperature dependence of isotherms in hazelnuts. This model will allow the description of water adsorption/desorption in porous media. The model is based on the Fick's law with water vapor pressure and temperature as driving potentials. For 1-dimensinal, non- isothermal model consists in the following equation solved for $\mathrm{p}$ :

$$
\rho_{0} \frac{\partial u}{\partial \varphi}\left(\frac{\frac{\partial p}{\partial t} p_{s}-p \frac{\partial p_{s}}{\partial t}}{p_{s}^{2}}\right)=D_{p} \frac{\partial^{2} p}{\partial x^{2}}+D_{T} \frac{\partial^{2} T}{\partial x^{2}}
$$

where $\rho_{0}$ is the dry porous material density, $u$ is the equilibrium moisture content, $p$ is the pressure, $p_{\mathrm{s}}$ is the saturation pressure, $t$ is time, $x$ is the coordinate, $\varphi$ is the ratio between the pressure and the saturation pressure, $D_{\mathrm{p}}$ is the water vapour diffusion coefficient with vapour pressure as a potential, also being a non-linear function of water vapour pressure (or moisture content $u$ or relative humidity $R H$ ) and temperature, $D_{\mathrm{T}}$ is the thermal moisture diffusion coefficient (a quantity which can be a complex function of both $u$ and T). An explicit finiteelement method was implemented in a spreadsheet. Air temperature and relative humidity are used as input parameters. The moisture content, moisture capacity, diffusion coefficient, temperature, saturation vapour pressure and vapour pressure fields are updated at each time step. The numerical tool was validated at the "Laboratorio di Misure Industriali" (LAMI) in the temperature range between $0{ }^{\circ} \mathrm{C}$ and $60{ }^{\circ} \mathrm{C}$. Qualitative transient results are shown in Figures 14 and 15 in terms of equilibrium moisture content as a function of the hazelnut radius for different times. In particular, the numerical simulations were performed considering two different relative humidity of the surrounded air equal to $20 \%$ (Figure 14) and $60 \%$ (Figure 15) and an equilibrium temperature equal to $30^{\circ} \mathrm{C}$. From the these results, we can see that the water accumulation starts from the outer surface of the hazelnut and then propagates inwards. In steady state conditions, a constant value of the moisture content is reached within the sample.

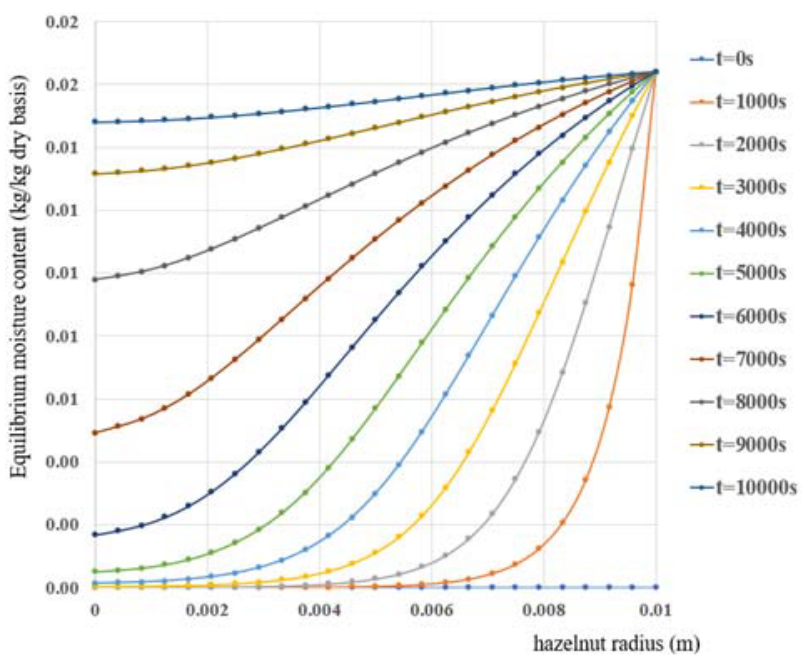

Fig. 14. Transient numerical results for equilibrium moisture content as a function of the hazelnut radius obtained for the conditions of $20 \%$ and $30{ }^{\circ} \mathrm{C}$. 


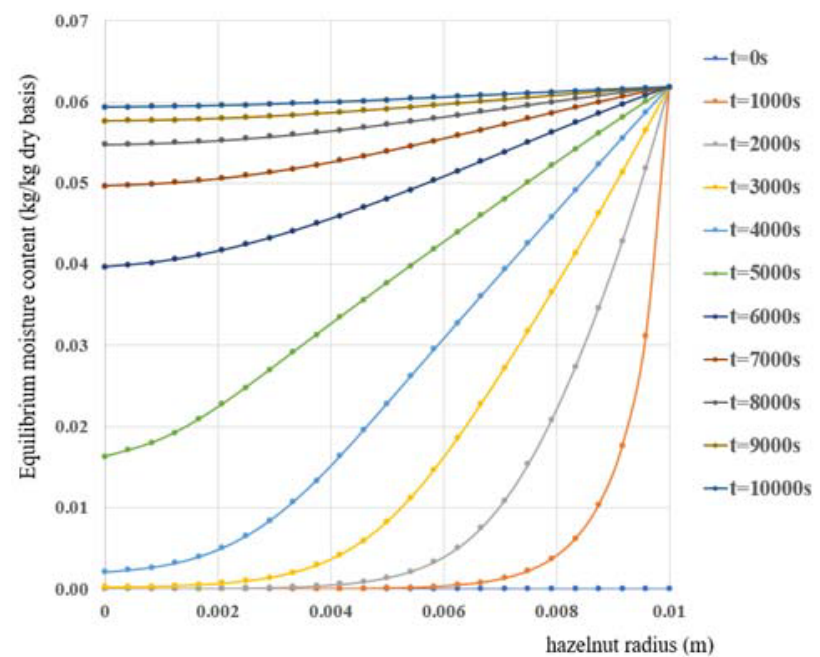

Fig. 15. Transient numerical results for equilibrium moisture content as a function of the hazelnut radius obtained for the conditions of $60 \%$ and $30{ }^{\circ} \mathrm{C}$.

\section{Humidity control in specific microbial transient processes}

Specific microbial transient processes (microbial growth) in pharmaceutical industry seemingly accumulate high humidity within small confined environment. Additionally, significant environmental shocks to bacteria from the transient exposure of the sample to different temperatures of agar may occur. Existing ambient control procedures prove to be insufficient because of apparent formation of unwanted moisture at specific locations, which effects read out of results at the end of incubation.

Condensation on the inside lid of Petri dishes can form whenever a temperature differential occurs. For example, when the temperature of the inside lid falls below the temperature due to surrounding air temperature, the condensate inside the Petri dish forms. This occurs most often when the temperature in a room fluctuates and falls, even by as little as $0.1^{\circ} \mathrm{C}$.

In order to measure small gradients of temperature and humidity, University of Ljubljana (UL) has designed and used a set of precision thermometers and hygrometers, which have minimized effect on the process. Both parameters were measured inside as well as outside the Petri dishes stacks. To analyse and reduce the unwanted condensation, different parameters were varied, such as the number of Petri dishes in the stack, the temperature of the incubator, the surrounding etc. Fig. 16 shows an example of the measurement setup.

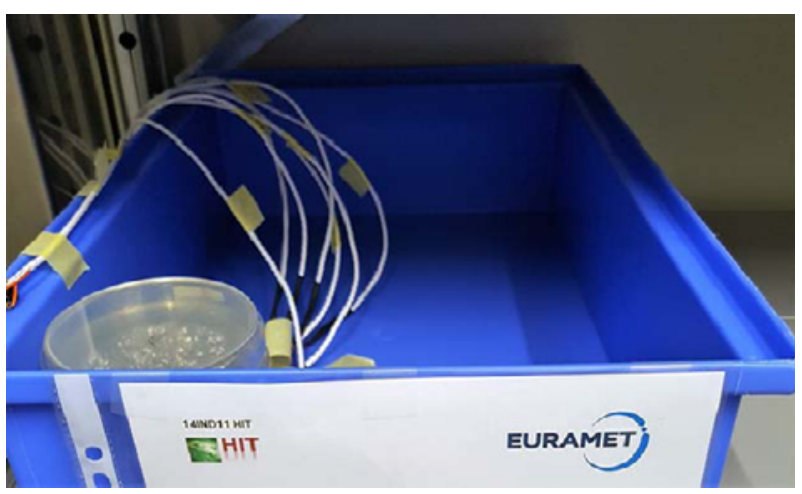

Fig. 16. A photograph showing a measurement setup for measuring small gradients of temperature and humidity in a Petri dish stack.

\section{Conclusions and discussion}

The HIT project is developing new and improved methods and techniques for humidity measurement and monitoring at high temperatures and under transient conditions. In this paper we reported advancements in developing calibration and test facilities for industrial hygrometers, performing tests to develop new calibration procedures for high temperatures or non-static conditions, developing a novel inline water activity measurement method and studying optimal humidity control in specific microbial transient processes.

The development of calibration facilities enabled starting tests for hygrometers at static conditions at temperatures up to $180{ }^{\circ} \mathrm{C}$ and pressures up to $0.6 \mathrm{MPa}$. For tests at non-static conditions, a new setup was designed and constructed with easy use in temperature test chambers. Using this equipment, tests of commercial humidity sensors with slower and faster humidity changes are ongoing. Combining stable humidity generation, dew-point measurement and continuous weighing a sample chamber in an oven enabled us to construct a new type measuring system for sorption isotherm. This and the developed transient tool for modelling temperature dependence of isotherms are key components of the new inline water activity measurement method. A measurement setup for determining small temperature and humidity gradients in a Petri dish stack were presented.

In addition to the work reported in this paper, HIT is developing a new direct Tunable Diode Laser Absorption Spectroscopic (dTDLAS) hygrometer for measuring humidity transients at high temperatures and a new type of field humidity calibrator.

This work is funded by the EMPIR initiative that is co-funded by the European Union's Horizon 2020 research and innovation programme and the EMPIR Participating States. 


\section{References}

1. M. Heinonen, L. Cavallarin, M. Dell'Isola, V. Ebert, V. Fernicola, E. Georgin, D. Hudoklin, J. Nielsen, P. F. Østergaard, A. Peruzzi, T. Pietari, S. Wagner, O. Werhahn, Metrology for humidity at high temperatures and transient conditions, International Congress of Metrology 15015 (2015)

2. S. Tabandeh, V. Fernicola, A Mass fraction humidity generator for specific humidity up to 0.5 $\mathrm{kg} / \mathrm{kg}$ and pressure up to $0.6 \mathrm{MPa}$, Abstracts. XIII Int. Symposium on Temperature and Thermal Measurements in Industry and Science TEMPMEKO 2016 (INTiBS, Kraków, 2016), pp. 124

3. S. Tabandeh, R. Gavioso, V. Fernicola, Validation of a generator for dew point temperature up to 0.5 $\mathrm{kg} / \mathrm{kg}$ and pressure up to $0.6 \mathrm{MPa}$ with microwave hygrometry, Abstracts. XIII Int. Symposium on Temperature and Thermal Measurements in Industry and Science TEMPMEKO 2016 (INTiBS, Kraków, 2016), pp. 194

4. R. Bosma, A. Peruzzi, Int J Thermophys 35:738747 (2014)

5. R. Bosma, A. Peruzzi, J. van Geel, Relative humidity at temperatures up to $180{ }^{\circ} \mathrm{C}$ and pressures up to 6 bar, Abstracts. XIII Int. Symposium on Temperature and Thermal Measurements in Industry and Science TEMPMEKO 2016 (INTiBS, Kraków, 2016), pp. 217 